\title{
Patient knowledge on stroke risk factors, symptoms and treatment options
}

This article was published in the following Dove Press journal: Vascular Health and Risk Management

\author{
Kashif Waqar Faiz ${ }^{1,2}$ \\ Antje Sundseth' \\ Bente Thommessen' \\ Ole Morten Rønning ${ }^{1,3}$ \\ 'Department of Neurology, Akershus \\ University Hospital, Lørenskog, \\ Norway; ${ }^{2}$ Health Services Research \\ Center, Akershus University Hospital, \\ Lørenskog, Norway; ${ }^{3}$ Institute of \\ Clinical Medicine, University of Oslo, \\ Oslo, Norway
}

Background: Public campaigns focus primarily on stroke symptom and risk factor knowledge, but patients who correctly recognize stroke symptoms do not necessarily know the reason for urgent hospitalization. The aim of this study was to explore knowledge on stroke risk factors, symptoms and treatment options among acute stroke and transient ischemic attack patients.

Methods: This prospective study included patients admitted to the stroke unit at the Department of Neurology, Akershus University Hospital, Norway. Patients with previous cerebrovascular disease, patients receiving thrombolytic treatment and patients who were not able to answer the questions in the questionnaire were excluded. Patients were asked two closed-ended questions: "Do you believe that stroke is a serious disorder?" and "Do you believe that time is of importance for stroke treatment?". In addition, patients were asked three open-ended questions where they were asked to list as many stroke risk factors, stroke symptoms and stroke treatment options as they could.

Results: A total of 173 patients were included, of whom $158(91.3 \%)$ confirmed that they regarded stroke as a serious disorder and 148 patients (85.5\%) considered time being of importance. In all, 102 patients $(59.0 \%)$ could not name any treatment option. Forty-one patients $(23.7 \%)$ named one or more adequate treatment options, and they were younger $(p<0.001)$ and had higher educational level $(p<0.001)$, but had a nonsignificant shorter prehospital delay time $(p=0.292)$.

Conclusion: The level of stroke treatment knowledge in stroke patients seems to be poor. Public campaigns should probably also focus on information on treatment options, which may contribute to reduce prehospital delay and onset-to-treatment-time.

Keywords: stroke, thrombolytic therapy, endovascular treatment, prehospital delay, stroke knowledge, stroke treatment options

\section{Introduction}

The emergence of intravenous thrombolysis with tissue plasminogen activator (tPA) and endovascular treatment with thrombectomy has increased the focus on stroke as a condition of urgency and emergency, as these treatments are limited by narrow time windows and the benefit decreases when onset-to-treatment-time (OTT) increases. ${ }^{1,2}$ In order to utilize these highly effective, but time-sensitive treatment options, prehospital delay and OTT have to be minimized so that the patients arrive at the emergency department within the time window and can be considered for tPA and/or thrombectomy.

Public information campaigns focus primarily on stroke symptom recognition, ${ }^{3,4}$ but patients who correctly recognize stroke symptoms do not necessarily seek attention
Correspondence: Kashif Waqar Faiz Department of Neurology, Akershus University Hospital, N-I478 Lørenskog, Norway

Fax +4767968132

Tel +4791682740

Email kashif.faiz@medisin.uio.no 
urgently. These campaigns have often had limited effect. ${ }^{5}$ One reason could be the lack of awareness of treatment options and the purpose of urgent hospitalization.

The aim of this study was to explore knowledge on stroke risk factors, symptoms and treatment options among acute stroke and transient ischemic attack (TIA) patients without previous cerebrovascular disease.

\section{Methods}

This study was part of a prospective project on prehospital delay and patient knowledge among stroke patients admitted to the stroke unit at the Department of Neurology, Akershus University Hospital, Norway, during a 1-year period (20092010). All patients in the catchment area with presumed stroke and TIA are admitted directly to the hospital's emergency department. Consecutive patients with acute ischemic stroke, intracerebral hemorrhage and TIA were included in this study $(n=440)$. Exclusion criteria were: patients who were not able to answer the questions in the questionnaire $(n=153)$, patients receiving thrombolytic treatment $(n=27)$ and patients with previous cerebrovascular disease $(n=87)$, the latter two because of knowledge bias. Details have been described elsewhere. ${ }^{6}$ No public campaigns regarding stroke were conducted before or during the study period. All patients were managed according to standard stroke guidelines.

Information was collected through a structured questionnaire which was completed within 72 hours after admission.

Patients were asked two closed-ended questions: "Do you believe that stroke is a serious disorder?" and "Do you believe that time is of importance for stroke treatment?". In addition, patients were asked three open-ended questions where they were asked to list as many stroke risk factors, stroke symptoms and stroke treatment options as they could.

Prehospital delay was defined as the time interval from symptom onset to hospital arrival. In wake-up strokes, the time of awakening was considered as the time of symptom onset. For patients with unknown/uncertain time of symptom onset (the patient unable to specify the exact time of onset because of, eg, reduced consciousness or aphasia), the time when they were witnessed by others was considered as the time of symptom onset. Education was dichotomized to either primary/lower secondary/upper secondary or higher educational level (university college/university).

The study was approved by the Norwegian Regional Committee for medical and health research ethics and by the hospital's local Data Protection Officer. All participants gave their oral informed consent.

\section{Statistical analysis}

Patient characteristics are presented as mean (SD) or median (interquartile range $[\mathrm{IQR}]$ ) values, depending on whether the variables were normally distributed. Comparisons between groups were performed with Mann-Whitney $U$ test for continuous variables and Pearson's $\chi^{2}$ test or Fisher's exact test (as appropriate) for categorical variables. A $p$ value $<0.05$ was considered statistically significant.

Statistical analysis was performed using IBM SPSS Statistics version 21.

\section{Results}

A total of 173 patients were included in the study. Mean age was 68.0 years (SD 13.8), and $63.6 \%$ were men. The distribution of cerebrovascular disease was: $120(69.4 \%)$ ischemic strokes, 7 (4.0\%) intracerebral hemorrhages and $46(26.6 \%)$ TIAs. Median prehospital delay was 4.6 hours (IQR 1.7-13.5), and 106 patients (61.3\%) were admitted by ambulance. Patient characteristics are presented in Table 1.

In all, 158 patients $(91.3 \%$ ) confirmed that they regarded stroke as a serious disorder and 148 patients $(85.5 \%)$ answered yes to the question of time being of importance for stroke treatment.

The 10 most mentioned stroke risk factors and stroke symptoms are listed in Tables 2 and 3, respectively. No patients mentioned higher age, male gender or prior cerebrovascular disease as risk factors.

Regarding acute stroke treatment (Table 4), 102 patients $(59.0 \%)$ could not name any treatment option. Twenty-five patients $(14.5 \%)$ named "anticoagulants/antithrombotic treatment/blood thinners" as a treatment option, while 12

Table I Patient characteristics

\begin{tabular}{ll}
\hline Characteristics & \\
\hline Men & $110(63.6)$ \\
Age (years) & $68.0(13.8)$ \\
NIHSS & $2(1-4)$ \\
Hypertension & $106(61.3)$ \\
Hyperlipidemia & $94(54.3)$ \\
Previous coronary disease & $49(28.3)$ \\
Diabetes mellitus & $25(14.5)$ \\
Current smoker & $44(25.4)$ \\
Higher educational level & $51(29.5)$ \\
Living alone & $53(30.6)$ \\
Time of onset & \\
$\quad$ Known & $113(65.3)$ \\
Wake-up & $47(27.2)$ \\
Unknown/uncertain & $13(7.5)$ \\
\hline
\end{tabular}

Notes: Categorical variables are presented as absolute values (percentages). Age is presented as mean (standard deviation), and NIHSS as median (interquartile range). Abbreviation: NIHSS: National Institutes of Health Stroke Scale. 
Table 2 Stroke symptom knowledge ( $\mathrm{N}=173)$

\begin{tabular}{ll}
\hline Symptoms & $\mathbf{n}(\%)$ \\
\hline Weakness in arm/leg & $105(60.7)$ \\
Speech difficulties & $87(50.3)$ \\
Facial drop & $56(32.4)$ \\
Vision difficulties & $11(6.4)$ \\
Dizziness & $8(4.6)$ \\
Reduced memory & $8(4.6)$ \\
Headache & $6(3.5)$ \\
Loss of balance & $6(3.5)$ \\
Loss of consciousness & $5(2.9)$ \\
Confusion & $2(1.2)$ \\
\hline
\end{tabular}

Table 3 Stroke risk factor knowledge $(N=173)$

\begin{tabular}{ll}
\hline Risk factors & $\mathbf{n ~ ( \% )}$ \\
\hline Smoking & $46(26.6)$ \\
Hypertension & $37(21.4)$ \\
Obesity/being overweight & $21(12.1)$ \\
Alcohol overuse & $20(11.6)$ \\
Diabetes & $15(8.7)$ \\
Poor/unhealthy diet & $14(8.1)$ \\
Cardiac disease & $13(7.5)$ \\
Lack of exercise & $13(7.5)$ \\
Heredity & $12(6.9)$ \\
Chronic obstructive pulmonary disease & $6(3.5)$ \\
\hline
\end{tabular}

Table 4 Stroke treatment option knowledge $(\mathrm{N}=173)$

\begin{tabular}{ll}
\hline Treatment & $\mathbf{n}(\%)$ \\
\hline Blood thinners & $25(14.5)$ \\
Rehabilitation/training & $2 I(12.1)$ \\
Thrombolytic/clot-dissolving treatment & $12(6.9)$ \\
Drug (undefined) given quickly & $8(4.6)$ \\
Physiotherapy & $5(2.9)$ \\
Drug (undefined) & $5(2.9)$ \\
Operation & $4(2.3)$ \\
Diet & $2(1.2)$ \\
Intravenous fluid & $1(0.6)$ \\
\hline
\end{tabular}

patients (6.9\%) named "intravenous thrombolytic therapy/ clot-dissolving treatment" as a treatment option. Eight patients mentioned "some kind of drug which must be given quickly", without being able to name the drug. In all, 41 patients $(23.7 \%)$ mentioned one or more of these treatment options, and they were significantly younger (59.5 [SD 13.1] versus 70.6 [SD 12.9] years, $p<0.001$ ) and had significantly higher educational level $(58.5 \%$ versus $41.5 \%, p<0.001)$, but had a nonsignificant shorter prehospital delay time (3.0 [IQR 1.8-11.5] versus 5.5 [IQR $1.6-13.5$ ] hours; $p=0.292$ ).

\section{Discussion}

In the present study, 1 in 11 acute stroke and TIA patients did not regard stroke as a serious disorder and 1 in 7 did not consider time being of importance for stroke treatment. In all, $6.9 \%$ of the patients could name intravenous thrombolytic therapy as a treatment option. Patients being able to name one or more relevant stroke treatment options were younger and had higher educational level. Interestingly, $59.0 \%$ of the patients were not able to name any treatment option.

Most studies on stroke knowledge have focused only on symptoms and risk factors, and not on knowledge of treatment options. ${ }^{7}$ Without specific knowledge on treatment options, and importantly, not knowing about the time sensitivity, it is unlikely that symptom recognition will automatically translate into action. Therefore, public campaigns should also emphasize that time is of great importance in order to utilize the most effective treatment options.

In an Italian telephone survey study with close-ended questions, $26.2 \%$ of the patients had knowledge of tPA treatment $^{8}$ and $15.0 \%$ of stroke unit availability. In a Canadian study comparing two cohorts (2010 and 2015), patients were asked about treatments that would prevent a stroke. ${ }^{9}$ The most common response was medication ( $87.9 \%$ in 2010 and $82.5 \%$ in 2015), and a significantly larger proportion identified exercise and stenting in 2015.

In a study from 2001 on acute stroke treatment knowledge, ${ }^{10} 21.0 \%$ mentioned blood clot-dissolving drugs or blood-thinning drugs and $12.1 \%$ mentioned blood pressure control, surgery, heart massage, other medications and natural therapies as treatment options. Interestingly, $66.9 \%$ could not identify any of these appropriate therapies. ${ }^{10}$ Although the study is not directly comparable to our study because of different populations (telephone interview participants versus acute stroke patients), both studies show that approximately only one in five patients know about blood clot-dissolving/ blood-thinning drugs, and that three in five are not able to name any treatment options. Importantly, although $85.5 \%$ of the patients in this study acknowledged time being of importance for stroke treatment, only $11.5 \%$ mentioned thrombolytic/clot-dissolving treatment or some kind of drug given quickly as a treatment option, underlining that few patients are aware of the reason for time being of importance.

The study has some limitations. This is a single-center study with a small sample size. The patients' stroke symptoms were not recorded; only the total National Institutes of Health Stroke Scale score was recorded. The patients' own symptoms could influence on their stroke symptoms knowledge. Another knowledge bias is information on treatment options gained after hospital admission, but before the questionnaire was completed (within 72 hours). In order to reduce this bias, patients with previous cerebrovascular disease and patients 
receiving thrombolytic treatment were excluded. On the other hand, the selection of patients means that the results cannot necessarily be generalized, and must thus be verified in a larger study sample from the general population.

This study was performed before the introduction of thrombectomy as standard treatment. Recognition of stroke as an emergency may have increased due to the publicity of this therapy.

In conclusion, this study shows that the level of stroke treatment knowledge in stroke patients seems to be poor. If these results can be verified in studies including an unselected population, it is reasonable to expect that future public campaigns also focus on information on treatment options, which may contribute to reduce prehospital delay and OTT.

\section{Disclosure}

The authors report no conflicts of interest in this work.

\section{References}

1. Lees KR, Bluhmki E, von Kummer R, et al. Time to treatment with intravenous alteplase and outcome in stroke: an updated pooled analysis of ECASS, ATLANTIS, NINDS, and EPITHET trials. Lancet. 2010;375(9727):1695-1703.
2. Fransen PS, Berkhemer OA, Lingsma HF, et al; Multicenter Randomized Clinical Trial of Endovascular Treatment of Acute Ischemic Stroke in the Netherlands Investigators. Time to reperfusion and treatment effect for acute ischemic stroke: a randomized clinical trial. JAMA Neurol. 2016;73(2):190-196.

3. Wall HK, Beagan BM, O’Neill J, Foell KM, Boddie-Willis CL. Addressing stroke signs and symptoms through public education: the Stroke Heroes Act FAST campaign. Prev Chronic Dis. 2008;5(2):A49.

4. Rasura M, Baldereschi M, Di Carlo A, et al; Promotion and Implementation of Stroke Care in Italy Project Working. Effectiveness of public stroke educational interventions: a review. Eur J Neurol. 2014;21(1):11-20.

5. Lecouturier J, Rodgers H, Murtagh MJ, White M, Ford GA, Thomson RG. Systematic review of mass media interventions designed to improve public recognition of stroke symptoms, emergency response and early treatment. BMC Public Health. 2010;10:784.

6. Faiz KW, Sundseth A, Thommessen B, Ronning OM. Prehospital delay in acute stroke and TIA. Emerg Med J. 2013;30(8):669-674.

7. Teuschl Y, Brainin M. Stroke education: discrepancies among factors influencing prehospital delay and stroke knowledge. Int J Stroke. 2010;5(3):187-208.

8. Baldereschi M, Di Carlo A, Vaccaro C, Polizzi B, Inzitari D; Promotion Implementation of Stroke Care in Italy Project Working Group. Stroke knowledge in Italy. Neurol Sci. 2015;36(3):415-421.

9. Metias MM, Eisenberg N, Clemente MD, et al. Public health campaigns and their effect on stroke knowledge in a high-risk urban population: a five-year study. Vascular. 2017;25(5):497-503.

10. Sug Yoon S, Heller RF, Levi C, Wiggers J, Fitzgerald PE. Knowledge of stroke risk factors, warning symptoms, and treatment among an Australian urban population. Stroke. 2001;32(8):1926-1930.
Vascular Health and Risk Management

\section{Publish your work in this journal}

Vascular Health and Risk Management is an international, peerreviewed journal of therapeutics and risk management, focusing on concise rapid reporting of clinical studies on the processes involved in the maintenance of vascular health; the monitoring, prevention and treatment of vascular disease and its sequelae; and the involvement of

\section{Dovepress}

metabolic disorders, particularly diabetes. This journal is indexed on PubMed Central and MedLine. The manuscript management system is completely online and includes a very quick and fair peer-review system, which is all easy to use. Visit http://www.dovepress.com/ testimonials.php to read real quotes from published authors. 